\title{
Modelling power flow in a hoist motor of a Rubber Tyred Gantry crane
}

\author{
Stefano Pietrosanti, Student Member, IEEE, William Holderbaum, Member, IEEE, \\ and Victor M. Becerra, Senior Member, IEEE
}

\begin{abstract}
A method for calculating the power demand of the hoist motor in Rubber-Tyred Gantry (RTG) cranes with nonparallel cables has been developed in order to measure the energy consumption in a typical lift cycle. From measurements taken at the Port of Felixstowe, it has been observed that the peculiar geometrical characteristics of the hoisting mechanism causes the power demand to increase with the container height in constant rotor speed conditions. The change in the angle of the hoisting ropes cause an increase in torque load and power consumption. By using information extracted from the crane's geometry, it has been possible to calculate the potential energy increase given the weight and vertical position of the container. The load torque on the hoist motor and the vertical speed of the mass have also been calculated, allowing for the modelling of the hoist motor power consumption when lifting containers with constant rotational speed. The proposed model has been compared to a constant power demand approximation, showing a higher accuracy for masses below $40 \mathrm{t}$.
\end{abstract}

Index Terms-Load flow analysis, Load modeling, Mathematical model, Mechanical systems, Power system modeling.

\section{INTRODUCTION}

$\mathbf{T}$ HE stacking of cargo containers inside shipping ports is handled by Rubber-Tyred Gantry (RTG) cranes, whose role is to lift containers from a terminal tractor and stack them in the port area waiting to be moved to a train or to a ship. RTG cranes are usually diesel-powered, account for a significant quantity of the energy consumption inside the port, and are a major contributor to greenhouse gases emissions [1]. Shipping ports are now considering reducing the energy consumption by increasing efficiency, improving operations, and recovering energy that is otherwise wasted. The hoist motor of a crane, with a power rating of up to $400 \mathrm{~kW}$, accounts for the majority of the consumed energy and offers opportunities to recover potential energy when lowering a container [2]. Modelling the power flow of this element is crucial for increasing the efficiency and enabling the utilization of stored energy via a control methodology.

The hoist geometry of a gantry crane has been historically modelled as a single rope lifting a mass whose dynamic equation were used to control the swinging [3]. Later, the research problem moved towards multi-cable spreaders with parallel cables [4], [5] which result in a constant power consumption in

S. Pietrosanti and W. Holderbaum are with the School of Systems Engineering, University of Reading, Reading, RG6 6AY, UK (e-mail: s.pietrosanti@pgr.reading.ac.uk; w.holderbaum@ reading.ac.uk).

V. M. Becerra was with the School of Systems Engineering, University of Reading, Reading RG6 6AY, UK. He is now with the School of Engineering, University of Portsmouth, Portsmouth PO1 3DJ, UK (e-mail: victor.becerra@port.ac.uk). the steady state phase with constant rotor speed. By analysing data acquired on RTG cranes at the Port of Felixstowe it was observed that the shape of the power waveform cannot be modelled only by calculating the power required to move a mass vertically at a constant speed, as it is shown in Figure 1, since the power demand increases in the phase when the container is being lifted at a constant motor speed. The same behaviour, reversed, also occurs when lowering the container. This is due to the peculiar geometry of the crane hoisting mechanism that uses non-parallel cables, causing an increase in power demand with container height because of the changing vertical angle of the ropes: when the container is at the top, the power consumption (per unit of time) is higher due to the different rope angle. In [6] the authors find that the vertical speed of the spreader changes with the angle between the ropes and the vertical line passing through it. In order to facilitate the suppression of oscillations [7], the crane in study is equipped with non-parallel ropes, resulting in a similar geometry as the one presented in this paper. The geometry of hoisting cables has been primarily studied with the objective to reduce sway and oscillations, while the benefits are also found when modelling the power demand of a hoist motor. Accounting for non-parallel cables and the resulting change in power demand in steady state can expand the work done on RTG cranes, including the control of energy storage [8], [9], particularly in works that explicitly assume constant power demand in steady state [2], [10], [11]. Due to the unexpected increase in power demand during the constant speed phase, it is difficult to implement a control strategy designed specifically for cranes with parallel cables to cranes with non-parallel ones. Other types of cranes present a similar distinctive trait where the power load changes during the lift, like harbor portal cranes which have been the subject of a study [12], but to date an exhaustive analysis of the power consumption of an RTG with non-parallel ropes is still missing.

This paper intends to address this problem by calculating the vertical speed and acceleration of the container when the hoist motor is rotating at a constant speed it is possible to accurately measure the mechanical power provided by the motor, assuming it is possible to measure the weight of the load. The output of the calculation can be compared with the measured electric power flow to validate the proposed model. It is then now possible to estimate the energy consumption and recovery for any container movement without the need of any measurement on board the crane. This paper is an updated version of [13]. 


\section{Methods}

Figure 2 shows the crane, manufactured by ZPMC, which is the objective of this research. The headblock mechanism, or spreader is suspended from eight ropes and it locks to containers allowing the safe displacement of the load. The hoist motor is connected to the hoist drum through a reducer with a ratio of $122.8: 1$, which is then attached to the eight wire ropes that pass across sheaves; the ropes are then connected to the headblock, each rope forming the same angle with respect to the vertical axis. For the purposes of calculating container position and speed, only one rope is considered in the simplified geometry (Figure 3), assuming that the ropes contribute evenly to transfer the forces (due to gravity and inertia) from the container to the hoist drum and that the mass is restricted to only move vertically. A logging device recorded the rotational speed of the hoist motor, as well as electric quantities including currents and voltages, with a sampling rate of $100 \mathrm{~Hz}$. The hoist motor is a 8-poles induction motor rated at $200 \mathrm{~kW}$. Controlled tests have been performed using containers of known mass which have been lifted and lowered while logging measurements. A total of 5 test were conducted with container of the following weights: 0, 5, 10, 25 and 40 tonne. Motor speed data have been used to calculate the mechanical power while the power consumption has been used to validate the model.

1) Kinematics: Defining $c(t)$ as the length of a hoist rope at time $t$ in meters, we assume that we only have the knowledge of the speed $\dot{c}(t)$, calculated as:

$$
\dot{c}(t)=-\frac{\omega_{\text {hoist }}(t)}{n_{\text {red }}} \frac{d_{\text {drum }}}{2 \pi}
$$

where $\omega_{\text {hoist }}[\mathrm{rad} / \mathrm{s}]$ is the angular speed of the hoist motor (known), $n_{\text {red }}=122.8$ is the gear ratio of the reducer and $d_{\text {drum }}=1.285[\mathrm{~m}]$ is the diameter of the drum; $\dot{c}(t)$ is integrated to obtain the value of the rope length at time $t$. From the crane's schematics the range of values of $c(t), b(t)$ and $\theta$ have been extracted and are presented in Table I. The initial condition $c(0)$ is unknown so it initially chosen from the range of admissible values and then during the integration of $\dot{c}(t)$ the value of $\theta$ is calculated at each interval verifying that it does not steps out of the boundaries, in which case the initial condition $c(0)$ is changed accordingly. The integration is then restarted and the cycle is repeated until a suitable initial condition is found.

From the geometry in Figure 3 it can be seen that the value of $c(t)$ is linked to the values of lengths $a$ and $b(t)$ :

$$
c(t)^{2}=a^{2}+b(t)^{2}
$$

The value of $a$ measured from the schematics is approximately $2.640 \mathrm{~m}$. Knowing that $c(t)>a \forall t$, the vertical position $b(t)$ is the following:

$$
b(t)=\sqrt{c(t)^{2}-a^{2}}
$$

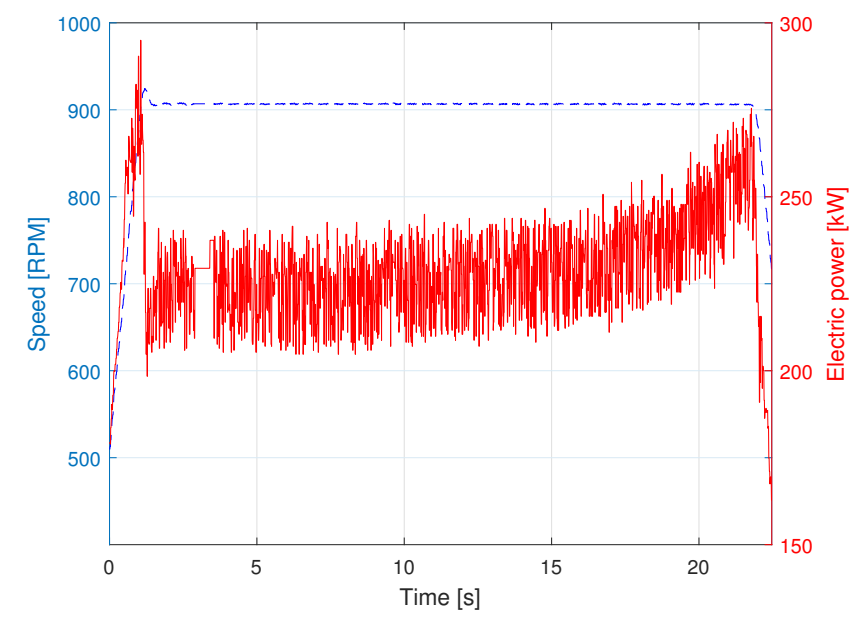

Fig. 1. Hoist motor speed (dashed blue line) and hoist motor power consumption (red) when lifting a $25 \mathrm{t}$ container.

TABLE I

MEASURED RANGES OF ROPE LENGTH $c$, CONTAINER VERTICAL POSITION $b$ AND ROPE ANGLE $\theta$.

\begin{tabular}{|c|cc|}
\hline$\forall t$ & Maximum height & Minimum height \\
\hline$c(t)$ & $4.200 \mathrm{~m}$ & $19.908 \mathrm{~m}$ \\
$b(t)$ & $3.643 \mathrm{~m}$ & $19.732 \mathrm{~m}$ \\
$\theta(t)$ & $\approx 39^{\circ}$ & $\approx 7.4^{\circ}$ \\
\hline
\end{tabular}

and speed of the container $\dot{b}(t)$ is then:

$$
\begin{aligned}
\dot{b}(t) & =\frac{d}{d t} \sqrt{c(t)^{2}-a^{2}} \\
& =\frac{1}{2 \sqrt{c(t)^{2}-a^{2}}} 2 c(t) \dot{c}(t) \\
& =\frac{c(t)}{\sqrt{c(t)^{2}-a^{2}}} \dot{c}(t) .
\end{aligned}
$$

Equation (5) implies that when the hoist motor speed is constant (and $\dot{c}(t)$ is constant) the vertical speed of the container changes with the length of the rope $c(t)$. The vertical speed of the container and the rope speed have a ratio which then depends on $c(t)$ :

$$
\frac{\dot{b}(t)}{\dot{c}(t)}=\frac{c(t)}{\sqrt{c(t)^{2}-a^{2}}} .
$$

The ratio in (6) is plotted in Figure 4 and it can be noticed that, with constant hoist motor speed, the vertical container speed $\dot{b}(t)$ increases as $c(t)$ decreases. The angle $\theta(t)$ varies as the container moves vertically, and it can be easily calculated from $a$ and $c(t)$ :

$$
\theta(t)=\arcsin \left(\frac{a}{c(t)}\right)
$$

The vertical acceleration can be calculated by further differentiating the speed:

$$
\begin{aligned}
\ddot{b}(t) & =\frac{d}{d t}\left(\frac{c(t) \dot{c}(t)}{\sqrt{c(t)^{2}-a^{2}}}\right) \\
& =\frac{c(t) \ddot{c}(t)+\dot{c}(t)^{2}}{\sqrt{c(t)^{2}-a^{2}}}-\frac{c(t)^{2} \dot{c}(t)^{2}}{\left(c(t)^{2}-a^{2}\right)^{3 / 2}}
\end{aligned}
$$




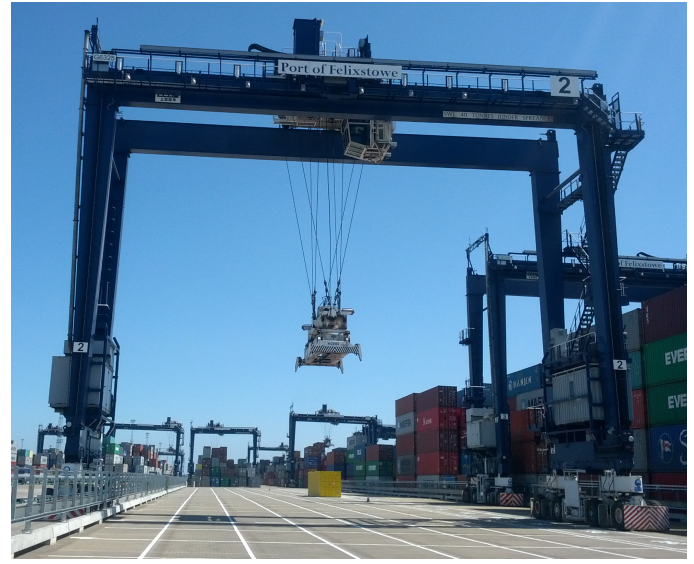

Fig. 2. Rubber-Tyred Gantry crane in use at the Port of Felixstowe.

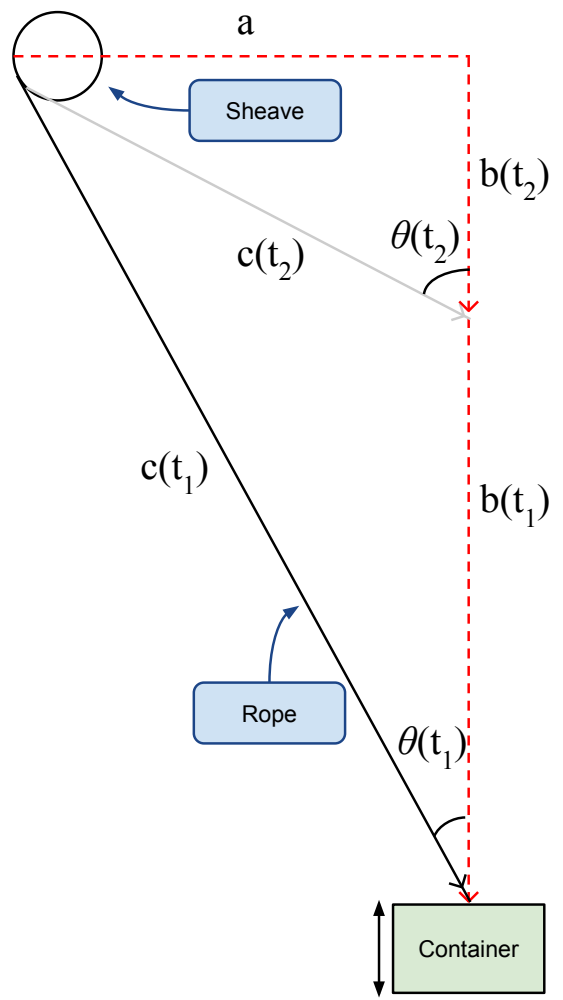

Fig. 3. Simplified 1-rope geometry of the hoisting mechanism. At time $t_{1}$ the container vertical position is lower so $b\left(t_{1}\right)$ and $c\left(t_{1}\right)$ are higher, therefore angle $\theta\left(t_{1}\right)$ is smaller. At time $t_{2}$ the vertical position is higher.

$$
=-\frac{c(t)\left(a^{2}-c(t)^{2}\right) \ddot{c}(t)+a^{2} \dot{c}(t)^{2}}{\left(c(t)^{2}-a^{2}\right)^{3 / 2}} .
$$

Limiting the calculations in the constant hoist motor speed phase, i.e. $\ddot{c}(t)=0$, Equation (9) can be simplified:

$$
\ddot{b}(t)=-\frac{a^{2} \dot{c}(t)^{2}}{\left(c(t)^{2}-a^{2}\right)^{3 / 2}} .
$$

The vertical acceleration, which varies depending on the height of the container, will be used to calculate the load torque on the motor.

2) Dynamics: To calculate the hoist motor power flow a load torque input is needed as well. As already stated, the

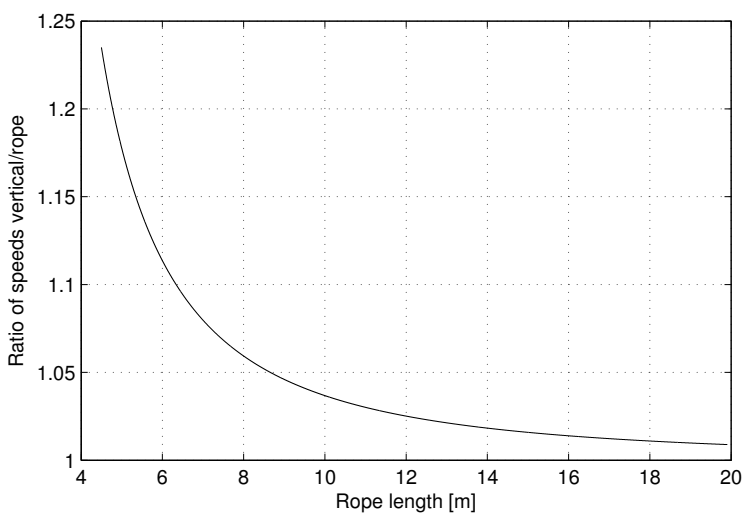

Fig. 4. Ratio between vertical speed and rope speed in function of rope length.

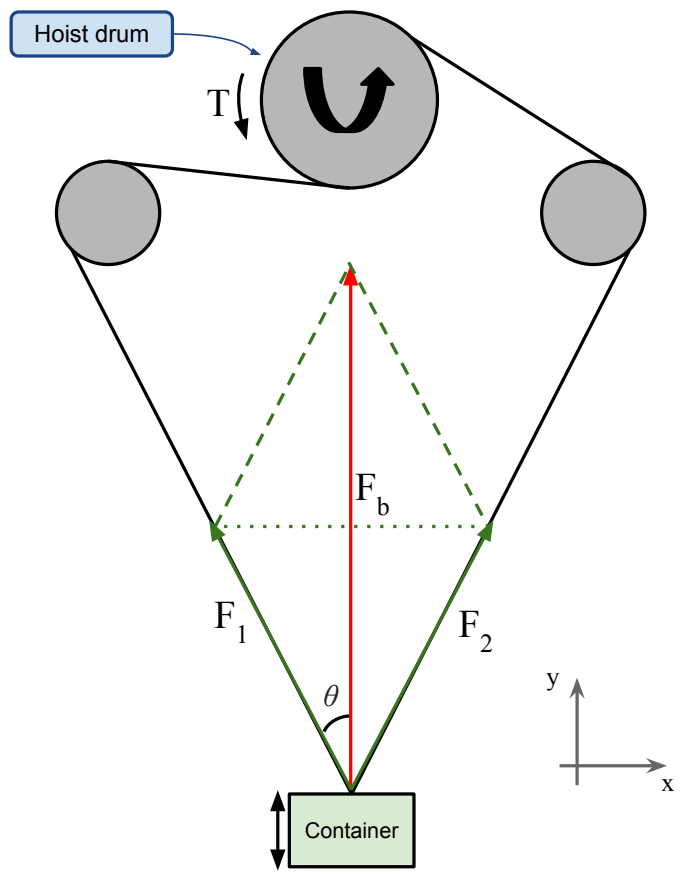

Fig. 5. Forces imposed by the hoist motor on the container when lifting.

power consumption increases with the container height when the speed is constant. The motor mechanical power $P_{m}$ is proportional to the torque $T$ times the rotational speed $\omega$; it is then evident that the variable that changes with height is the torque. In Figure 5 it can be seen that a torque $T$ is applied to the hoist drum causing it to lift the container by applying equal forces $\vec{F}_{1}$ and $\vec{F}_{2}$ whose sum results in the vector $\vec{F}_{b}$. By calculating the magnitude of $\vec{F}_{b}$ and the value of the angle $\theta$ it is possible to calculate the torque which the motor needs to apply to the drum (of known diameter). The vector $\vec{F}_{b}$ is the sum of two symmetrical components:

$$
\overrightarrow{F_{b}}=\vec{F}_{1}+\vec{F}_{2}
$$

and the modulus depends on the vertical components as the horizontal components cancel out:

$$
\left|\vec{F}_{b}\right|=F_{1, y}+F_{2, y}=2 F_{1, y}
$$


with $F_{n, y}$ being the component on the y-axis of $\vec{F}_{n}$. The component $F_{1, y}$ can be calculated from the values of $\theta(t)$, $a$ and $c(t)$ (from Figure 3):

$$
\begin{gathered}
\sin (\theta(t))=\frac{a}{c(t)} \\
\cos (\theta(t))=\sqrt{1-\frac{a^{2}}{c(t)^{2}}} \\
F_{1, y}(t)=\left|\vec{F}_{1}(t)\right| \cos (\theta(t))=\left|\vec{F}_{1}(t)\right| \sqrt{1-\frac{a^{2}}{c(t)^{2}}} .
\end{gathered}
$$

The magnitude of the force vector $\vec{F}_{1}$ that needs to be applied to generate a vertical force $\vec{F}_{b}$ on the container is then:

$$
\left|\vec{F}_{1}(t)\right|=\frac{\left|\vec{F}_{b}(t)\right|}{2 \sqrt{1-\frac{a^{2}}{c(t)^{2}}}} .
$$

At rest, the only force that the hoist motor needs to compensate is gravity. However, when the container is moved the sum of forces acting on the mass is:

$$
\begin{gathered}
\vec{F}=\left(m_{s}+m_{c}\right)\left(\vec{g}+\ddot{b}(t) \overrightarrow{u_{y}}\right)+\vec{F}_{b}=\overrightarrow{0} \\
\left|\vec{F}_{b}\right|=\left(m_{s}+m_{c}\right)(|\vec{g}|+\ddot{b}(t))
\end{gathered}
$$

where $m_{s}=13000$ is the mass (in $\mathrm{kg}$ ) of headblock and spreader, $m_{c}$ is the mass of the container, $\vec{g}$ is the acceleration given by gravity, $\overrightarrow{u_{y}}$ is the unit vector in the positive y direction and $\ddot{b}(t)$ is the instantaneous vertical acceleration imposed by the hoist motor as calculated in (10). Given the diameter $d_{d r u m}$ of the drum, the torque $\left|\vec{T}_{d}\right|$ that needs to be applied to the drum is then:

$$
\left|\vec{T}_{d}\right|=\left|\vec{F}_{b}\right| \frac{d_{d r u m}}{2}
$$

and, taking into account the presence of a reducer with ratio $n_{\text {red }}$, the hoist motor must provide the following instantaneous load torque $T$ :

$$
T(t)=\frac{1}{n_{\text {red }}} \frac{\left(m_{s}+m_{c}\right)\left(g+a_{c}(t)\right)}{\sqrt{1-\frac{a^{2}}{c(t)^{2}}}} \frac{d_{d r u m}}{2} .
$$

When the container is at rest, $\ddot{b}(t)=0$ and the only acceleration acting on the container is gravity:

$$
T(t)=\frac{d_{\text {drum }}}{2 n_{\text {red }}} \frac{g\left(m_{s}+m_{c}\right)}{\sqrt{1-\frac{a^{2}}{c(t)^{2}}}} .
$$

The calculated torque and speed values can be validated by comparing them to the measurement of consumed electric power.

\section{RESULTS AND DISCUSSION}

Measurements taken on a crane at the Port of Felixstowe were used to extract the container speed and acceleration. The hoist motor rotational speed was used to calculate the hoist rope position and speed using equation (1), as well as torque using equation (12).

Figure 6 shows the results of calculating the position of the container during a controlled test of hoist movement with a

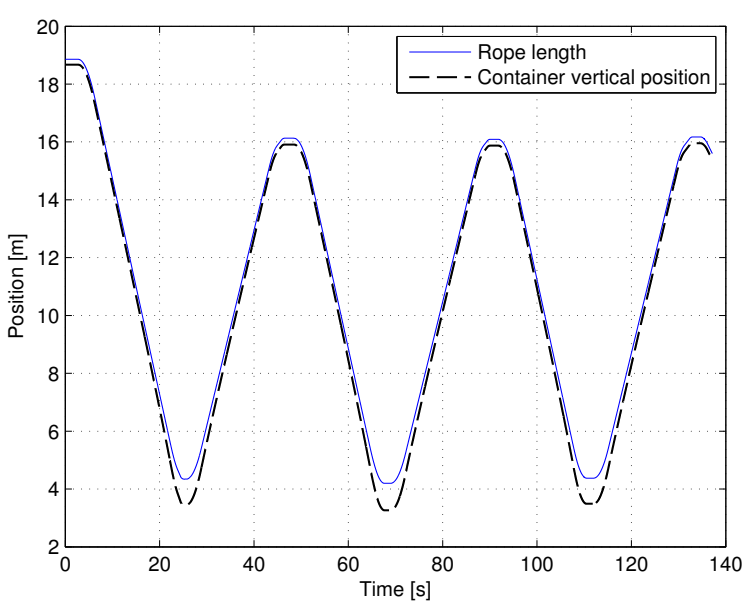

Fig. 6. Calculated rope position and container vertical position (origin at the headblock) for a controlled test when hoisting a $10 \mathrm{t}$ container.

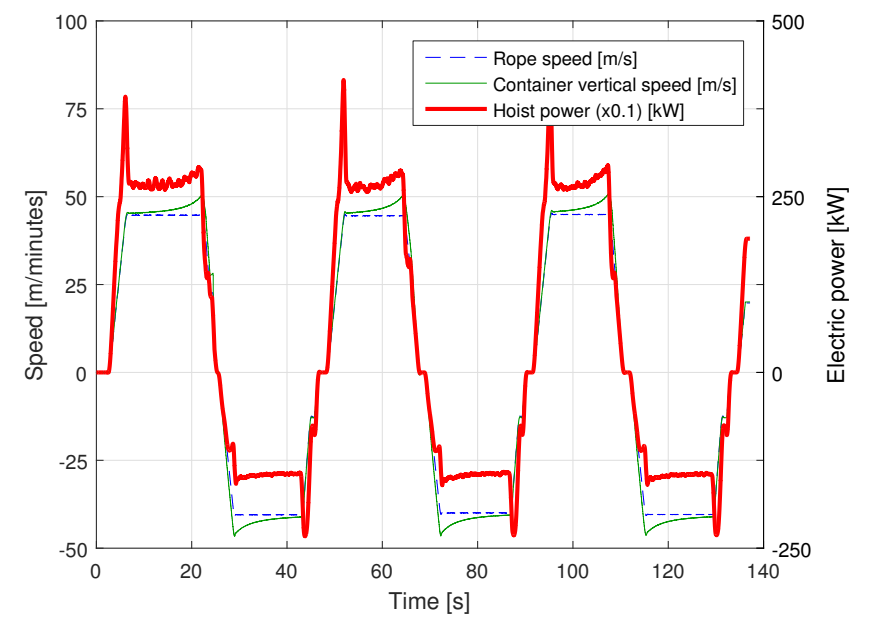

Fig. 7. Rope and container speed superimposed to the power flow of the hoist motor when lifting and lowering a $10 \mathrm{t}$ container. Note the lower power magnitude when the motor is regenerating (negative speed).

10 t container, using (1) and integrating. Figure 7 shows the speeds calculated by differentiating the vertical position; it can be noticed that the speeds are very close to the rated value of 50 meters per minute (provided by the manufacturer); it can also be verified that the power flow in the hoist motor (in red) changes with the calculated vertical position, albeit with constant motor speed and load mass.

From (13), the torque $T_{\text {rest }}$ that a hoist motor needs to provide to keep a 10 tonne container at rest can be calculated as:

$$
\begin{aligned}
T_{\text {rest }} & =\frac{d_{\text {drum }} g\left(10+m_{s}\right)}{2 n_{\text {red }}} \\
& =\frac{9.81^{\mathrm{m}} / \mathrm{s}^{2} \cdot(10 \mathrm{e} 3 \mathrm{~kg}+13 \mathrm{e} 3 \mathrm{~kg}) \cdot 1.285 \mathrm{~m}}{2 \cdot 122.8} \\
& =1180 \mathrm{Nm} .
\end{aligned}
$$

Given the values of $\dot{c}(t)$ (and, by integration, $c(t)$ ) it was possible to apply (12) in order to calculate the load torque on the motor. The result is shown in Figure 8. The gray signal of the calculated torque is highly sensible to noise in 


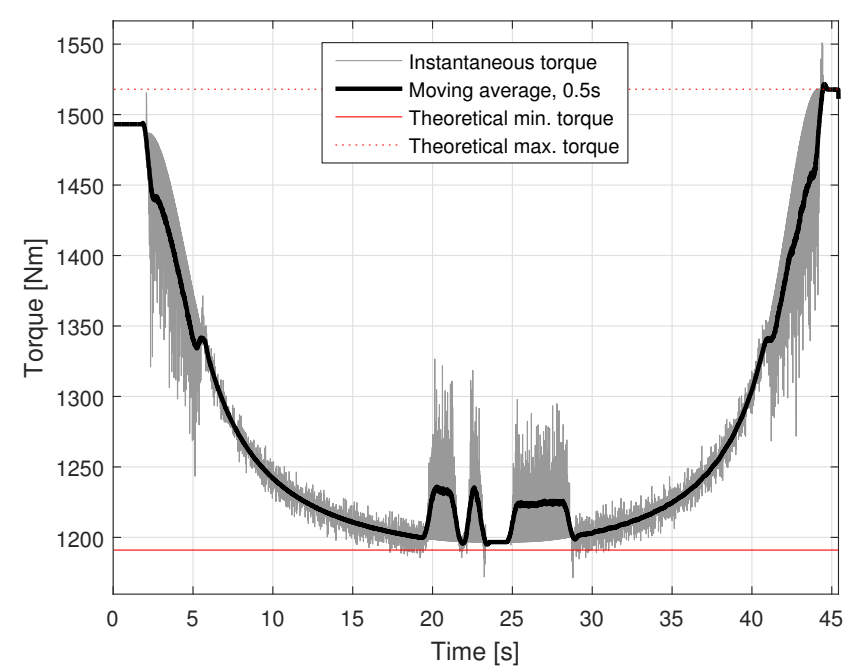

Fig. 8. Load torque on the hoist motor calculated from mass and acceleration of the container and the geometry of the system. A $10 \mathrm{t}$ container is lowered (first 23 seconds) and later it is raised again to approximately the same height. The boundaries (in red) are the theoretical maximum and minimum values for the calculated torque.

the hoist rotor speed measurement; for this reason it is also shown the moving average of the torque value. To mitigate the noise problem it is possible to use a constant motor speed as the input; in this work it was chosen to use the measurement in order to show the accuracy of the calculation. Assuming no vertical acceleration beside gravity and rope length spanning the whole admissible range, equation (12) indicates an expected torque range of approximately 1191 to $1518 \mathrm{Nm}$ during the whole movement, confirming the validity of the results shown in Figure 8.

Instantaneous power consumption of the hoist motor when lifting containers of known masses has been recorded at the Port of Felixstowe and it can be used to validate the results. The electric power consumption of an induction motor increases with the mechanical power required to lift the containers, the latter being the multiplication between the load torque $T(t)$, and rotational speed $\omega(t)$ :

$$
P_{m}(t)=\omega(t) T(t)
$$

where $P_{m}(t)$ is the instantaneous mechanical power that the motor need to produce in order to move a container at a certain speed, excluding electrical losses and mechanical losses. By dividing the losses into three categories, it is possible to link the mechanical power $P_{m}(t)$ to the electric power $P_{e}(t)$ :

$$
P_{m}(t)=a P_{e}(t)+b_{1} \omega(t)+b_{2}+\epsilon(t)
$$

where $a$ is a multiplicative factor that accounts for losses which depend on the electric power magnitude (e.g. copper losses), $b_{1}$ is linked to losses which depend on the speed (e.g. friction) and $b_{2}$ is a bias term which does not depend on speed or power. The function $\epsilon(t)$ includes the mechanical power modelling error and the higher-order approximations of the losses. By comparing the norm of $\epsilon(t)$ of the proposed model with respect to a constant-power approximation it is possible to demonstrate the validity of the model. The values of the
TABLE II

VALUES CHOSEN FOR APPROXIMATING THE CONVERSION LOSSES FROM MECHANICAL TO ELECTRIC POWER.

\begin{tabular}{|c|cc|}
\hline & Proposed model & Constant power approximation \\
\hline$a$ & 0.62 & 0.49 \\
$b_{1}$ & -0.28 & -0.33 \\
$b_{2}$ & 47.44 & 77.25 \\
\hline
\end{tabular}

TABLE III

RMSD VALUES OF THE PROPOSED MODEL AND THE CONSTANT POWER APPROXIMATION.

\begin{tabular}{|c|cc|}
\hline Weight & Proposed model & Constant power approximation \\
\hline $0 \mathrm{t}$ & 65.92 & 106.98 \\
$5 \mathrm{t}$ & 97.71 & 98.85 \\
$10 \mathrm{t}$ & 95.59 & 171.19 \\
$25 \mathrm{t}$ & 155.35 & 222.62 \\
$40 \mathrm{t}$ & 339.27 & 175.75 \\
\hline
\end{tabular}

parameters $a, b_{1}$ and $b_{2}$, shown in Table II, have been chosen as to minimize the normalized Root-Mean-Square Deviation (RMSD) $\|\epsilon(t)\|_{2}$ of both the model and the approximation.

The resulting values of $\|\epsilon(t)\|_{2}$ for each test are presented in Table III, and Figure 9 shows examples of the proposed model output compared with the approximation. It can be seen that the only test in which the proposed model has a worse performance than the approximation is the 40 tonne container, as reflected by both the high error (Table III) and profile (shown in Figure 9d). In the remaining tests the RMSD is lower than the approximation and the model tracks the power demand. The noisy output of the proposed model is due to the hoist rotor speed measurements and the calculations which accentuated the issue. As already stated in Section III, using constant speeds as inputs for the model instead of measurements will eliminate this problem.

\section{CONCLUSION}

Measurements of the power flow in a hoist motor of a Rubber-Tyred Gantry crane showed an unexpected increase in power consumption in constant speed movement. By analysing the geometry of the crane it had been possible to model the mechanical power required by a hoist motor to lift a container, including the variations in load torque and accelerations due to the geometry of the crane. A comparison of the model output and measurements taken at the Port of Felixstowe showed high model accuracy for loads up to $25 \mathrm{t}$. These results can lead to a more accurate model of the power flow of the hoist motor and the crane itself, allowing for the analysis of the energy consumption and the study of recoverable energy when lowering a container of known weight. A thorough knowledge on the amount of energy used in each lift may lead to energy savings techniques, including the use of energy storage, that can reduce the impact on the environment and the fuel cost of one of the most energy-intensive industries which is cargo container handling.

Future research could include the modelling of the dynamics of the electrical system, including the inevitable losses, leading 


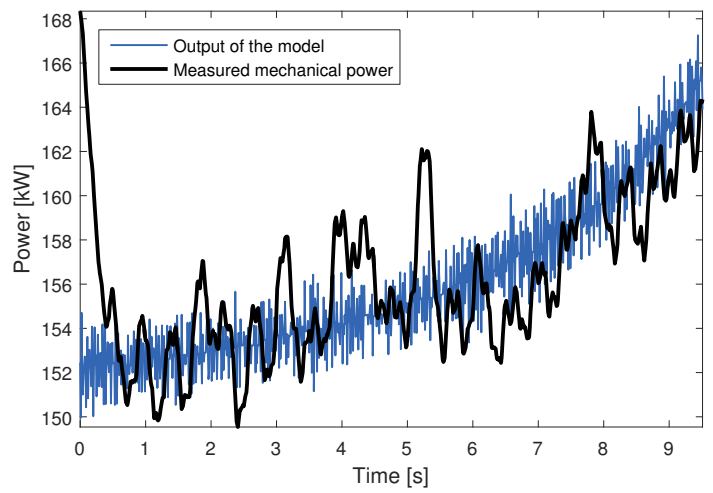

(a) $5 \mathrm{t}$ container

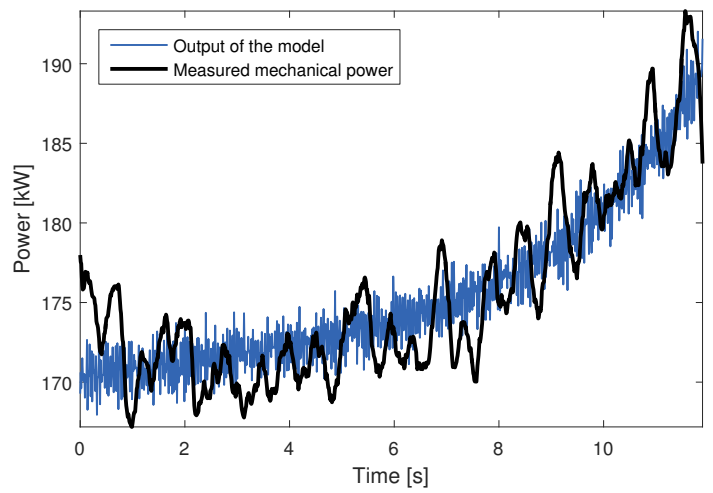

(b) $10 \mathrm{t}$ container

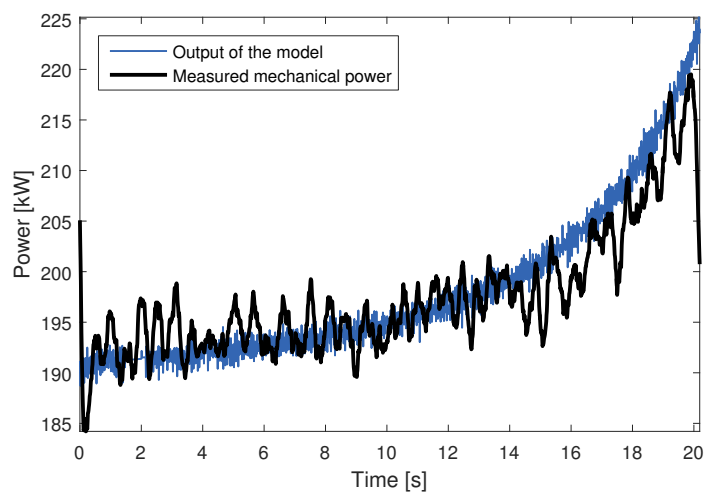

(c) 25 t container

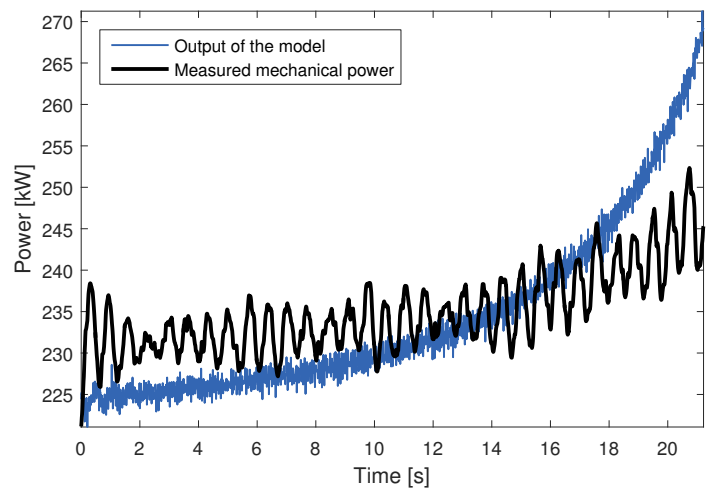

(d) $40 \mathrm{t}$ container

Fig. 9. Measured mechanical power compensated for losses (in black) compared to the output of the proposed model (in light blue). to a more accurate representation of the power consumption of the main electric motor.

\section{ACKNOWLEDGEMENT}

This research has been possible thanks to the data shared by the Port of Felixstowe and the help of their engineers. This research is part of the SUSPORTS project funded by Climate KIC.

\section{REFERENCES}

[1] "GREEN Technologies and Eco-Efficient Alternatives for CRANES and Operations at Port Container Terminals." [Online]. Available: http://www.greencranes.eu/GENERALPRESENTATION.pdf

[2] M. M. Flynn, P. McMullen, and O. Solis, "High-speed flywheel and motor drive operation for energy recovery in a mobile gantry crane," Conference Proceedings - IEEE Applied Power Electronics Conference and Exposition - APEC, pp. 1151-1157, 2007.

[3] B. DAndréa-Novel and J. Lévine, "Modelling and Nonlinear Control of an Overhead Crane," in Robust Control of Linear Systems and Nonlinear Control. Boston, MA: Birkhäuser Boston, 1990, pp. 523-529.

[4] L. Morrish, M. P. Cartmell, and A. J. Taylor, "Geometry and kinematics of multicable spreader lifting gear," Proceedings of the Institution of Mechanical Engineers, Part C: Journal of Mechanical Engineering Science, vol. 211, no. 3, pp. 185-194, 1997.

[5] M. P. Cartmell, L. Morrish, and A. J. Taylor, "Dynamics of spreader motion in a gantry crane," Proceedings of the Institution of Mechanical Engineers, Part C: Journal of Mechanical Engineering Science, vol. 212, no. 2, pp. 85-105, jan 1998.

[6] D.-H. Kim, J.-W. Lee, K.-T. Park, and J.-H. Oh, "Closed-form kinematic solution of a non-parallel cable reeving crane system," Proceedings of the Institution of Mechanical Engineers, Part C: Journal of Mechanical Engineering Science, vol. 217, no. 2, pp. 257-269, jan 2003.

[7] D.-H. Kim and J.-W. Lee, "Model-based PID control of a crane spreader by four auxiliary cables," Proceedings of the Institution of Mechanical Engineers, Part C: Journal of Mechanical Engineering Science, vol. 220, no. 8, pp. 1151-1165, aug 2006.

[8] D. Bayasgalan, J. S. Ryu, Y. M. Choi, S. H. Lee, D. H. Han, Y. J. Lee, H. R. Choi, and G. H. Choe, "Improved RTGC system with voltage compensation converter to reduce fuel consumption," 2012 IEEE Energy Conversion Congress and Exposition (ECCE), pp. 34-40, Sep. 2012.

[9] S. Kim and S. Sul, "Control of rubber tyred gantry crane with energy storage based on supercapacitor bank," Power Electronics, IEEE Transactions on, vol. 21, no. 5, pp. 1420-1427, 2006.

[10] F. Baalbergen, P. Bauer, and J. Ferreira, "Energy Storage and Power Management for Typical 4Q-Load," IEEE Transactions on Industrial Electronics, vol. 56, no. 5, pp. 1485-1498, may 2009.

[11] D. Iannuzzi, L. Piegari, and P. Tricoli, "Use of supercapacitors for energy saving in overhead travelling crane drives," in 2009 International Conference on Clean Electrical Power. IEEE, jun 2009, pp. 562-568.

[12] H. Liu, W. Mi, N. Zhao, and Y. Feng, "Modeling and Simulating the Operation of the Harbor Portal Crane," Journal of Coastal Research, vol. 73, no. 73, pp. 89-94, mar 2015.

[13] S. Pietrosanti, W. Holderbaum, and V. M. Becerra, "Modelling power flow in a hoist motor of a Rubber Tyred Gantry crane," in 2015 IEEE Industry Applications Society Annual Meeting. IEEE, oct 2015, pp. $1-6$.

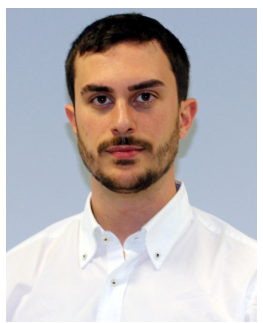

Stefano Pietrosanti Stefano Pietrosanti (S'15) received the BSc and MSc degrees in Automation Engineering at the University of Rome Tor Vergata, Rome, Italy, in 2009 and 2012, respectively.

$\mathrm{He}$ is currently pursuing a $\mathrm{PhD}$ at the School of Systems Engineering in the University of Reading where he is working on the SUSPORTS project. His current research interests focus on control of energy storage systems, analysis of power consumption, and system modellings of container cranes. 


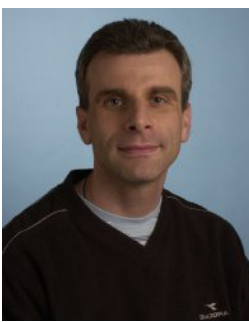

William Holderbaum Professor William Holderbaum (M'01) received the $\mathrm{PhD}$ degree from the University of Lille, Lille, France, in 1999. He was a Research Assistant at the University of Glasgow, Glasgow, U.K., from 1999 to 2001. He joined the University of Reading, Reading, UK, in 2001 as Lecturer and then as a senior lecturer/associate professor.

Currently, he is a Professor of Mathematics and Engineering in the School of Systems Engineering. His research interests are in control theory and its applications. These are mainly focused on geometric control theory in particular Hamiltonian systems and optimisation problems. This research uses the mathematical engineering skills in order to model and control energy storages in network with applications to industries (Port of Felixstowe and Scottish Southern Energy). Professor Holderbaum has also other research interest in control with application to rehabilitation engineering, energy reduction, smart grid, multiple agent systems, fuel cells, power generation, and autonomous vehicles.

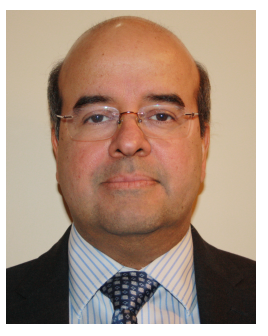

Victor M. Becerra Professor Victor Becerra (SM'03) completed his BEng in Electrical Engineering at Simon Bolivar University, Venezuela, in 1990, and his $\mathrm{PhD}$ in Control Engineering from City University, London, in 1994 . He is currently a Professor of Power Systems Engineering at the School of Engineering, University of Portsmouth, UK. Between 1989 and 1991, he was employed as an electrical engineer at C.V.G. Edelca, Caracas, Venezuela. Between 1994 and 1999 he was a postdoctoral research fellow the Control Engineering Research Centre at City University, London. During the period between 2000 and 2015 he was an academic at the School of Systems Engineering, University of Reading, UK.

His current research interests consider a range of issues related to electrical power systems, as well as the methods and applications of automatic control. These interests include control of power systems, energy storage and its integration to power grids, computational intelligence for smart grids, computational optimal control, nonlinear control, state estimation, and robotics. During his career he has received research funding from the EPSRC, the Royal Academy of Engineering, the European Space Agency, the Technology Strategy Board, the EU, and UK industry. 